\title{
Problèmes hydrauliques liés à la récupération d'hydrocarbures en mer
}

\author{
Hydraulic problems in offshore recovery \\ of hydrocarbons
}

\author{
Marc Darras \\ Département Laboratoire National d'Hydraulique EDF \\ Direction des Etudes et Recherches
}

Nous nous proposons de faire une synthèse des recherches dans le domaine hydraulique concernant le confinement et la récupération d'hydrocarbures en mer. Nous prendrons soin de souligner les conséquences pratiques des résultats des recherches actuelles et d'évaluer leur intérêt pour les recherches futures.

La présentation, en raison de sa brièveté, ne saurait être exhaustive et pourra sembler schématique sur certains points à des spécialistes. On trouvera en fin de texte une bibliographie détaillée.

La réalisation de matériel efficace de confinement - terme employé ici pour élargir le concept de barrage et de récupération nécessite une bonne compréhension du comportement relatif de l'eau, de l'huile et du matériel. Dès l'origine de ces études, à la fin des années 60 , on a conçu d'étudier séparément trois aspects qui apparaissent simultanément dans la nature :

- le comportement d'une nappe d'huile de dimension finie flottant sur un écoulement d'eau, et soumise à un cisaillement à l'interface eau-huile ;

- l'écoulement autour des barrages ou vers les appareils récupérateurs ;

- l'influence de la houle sur ces deux phénomènes.

\section{FORME D'UNE NAPPE D'HUILE}

La forme d'une nappe d'huile a certainement reçu le plus d'attention, et est, d'un point de vue théorique, sûrement la plus intéressante à court terme, car la plus abordable.

\section{Forme dans le courant}

\section{Une théorie simple}

La forme est définie essentiellement par l'équilibre entre les forces de pression hydrostatique dans la nappe - où les vitesses sont très faibles à cause de la viscosité et le frottement à l'interface eau-huile (Figure 1).
En prenant un coefficient de frottement $C_{f}$ constant, le profil est en racine carrée de la distance à partir de l'extrémité amont de la nappe d'huile (Cross et Hoult).

$$
\frac{g \Delta}{U^{2}} h=C_{f}^{1 / 2}\left(\frac{g \Delta x}{U^{2}}\right)^{1 / 2}
$$

avec

$$
\Delta=\frac{\rho \text { eau }-\rho \text { huile }}{\rho \text { eau }}
$$

avec $C_{f}$ de l'ordre de 0,01 à 0,005 . Ce résultat se trouve assez bien confirmé par l'expérience (Fig. 2).

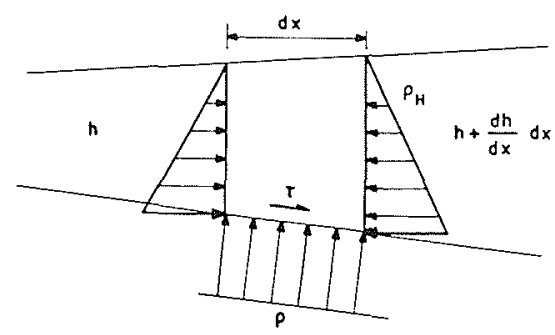

Figure 1 - Equilibre d'une tranche d'huile.

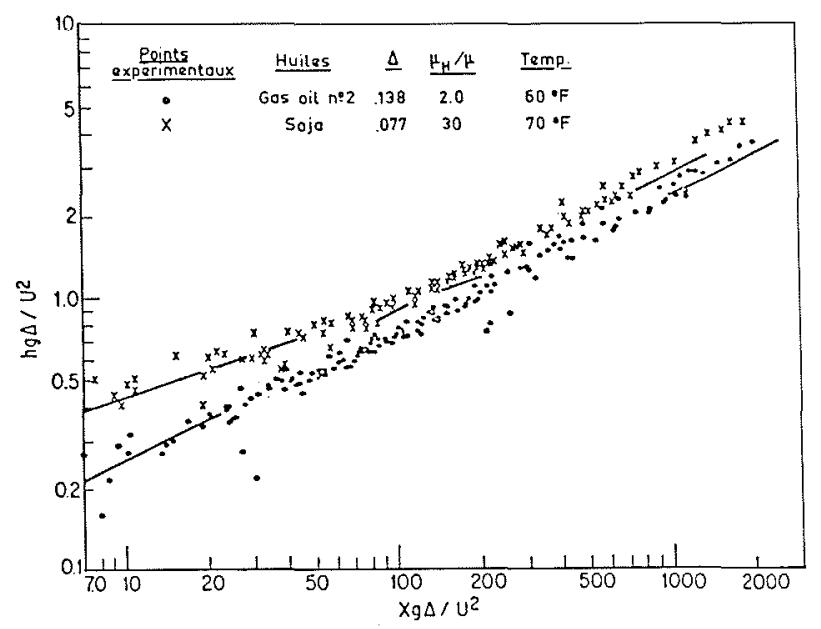

Figure 2 - Comparaison entre des expériences et la théorie d'après Cross et Hoult. 


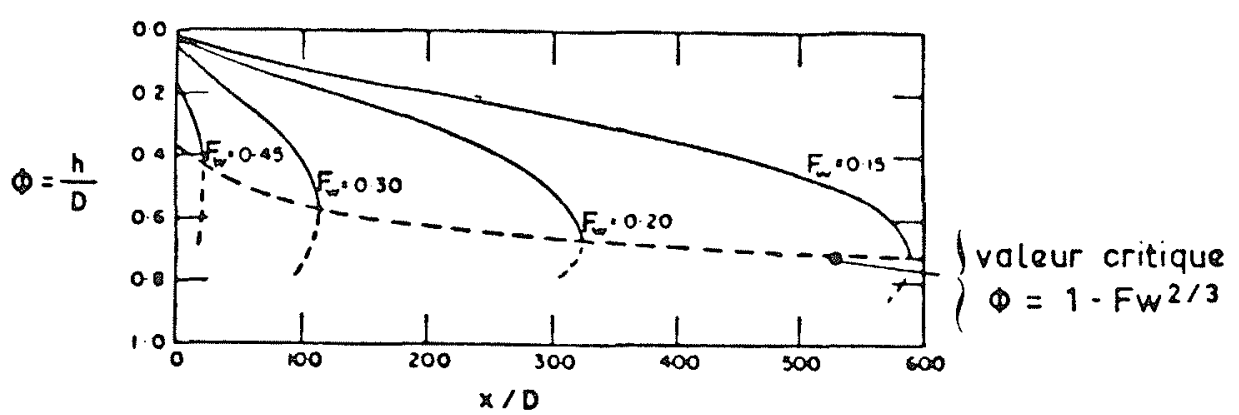

Figure 3 - Forme de la nappe pour différents nombres de Froude $F w, \Delta=0,15, f=0,015, f b=0,030$ d'après Wilkinson.

\section{Influence de la profondeur}

En profondeur réduite, la pression dynamique dans l'eau peut jouer un rôle en raison des accélérations possibles de cet écoulement, confiné entre l'interface et le fond (Wilkinson) (Fig. 3)

Bien évidemment ces deux théories coincident tant que l'épaisseur d'huile reste faible (Fig. 4).

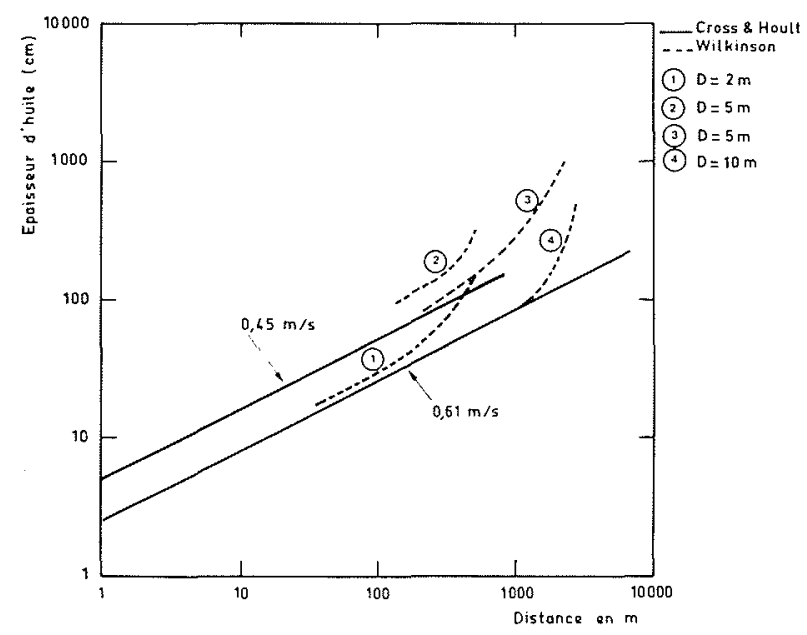

Figure 4 - Comparaison des épaisseurs en profondeur infinie (Cross et Hoult) au résultat obtenu en profondeur finie (Wilkinson).

Dans ces conditions on obtient une valeur critique de l'épaisseur qui ne peut être dépassée (Wilkinson).

$$
\frac{h}{D}=1-\frac{U^{2 / 3}}{\sqrt{\Delta g D}}
$$

C'est un auto-soutirage, mais nous aurons l'occasion de revenir sur ce point.

\section{Quelques écarts par rapport à la théorie simple}

Par rapport à la forme lisse que donne la seule prise en compte du frottement, l'interface eau-huile est le siège de nombreuses perturbations. Dès que la vitesse eau-huile dépasse approximativement $0,10 \mathrm{~m} / \mathrm{s}$. l'interface est parcourue par des ondes de gravité de longueur d'ondes "assez courte $(\sim 15 \mathrm{~cm})$ ". Lorsque la vitesse augmente, le bord de la nappe s'incurve de plus en plus pour finalement former une bosse frontale pour une vitesse voisine de $0,20 \mathrm{~m} / \mathrm{s}$. Enfin, à partir de vitesses de l'ordre de $0,40 \mathrm{~m} / \mathrm{s}$, des gouttelettes d'huile sont arrachées au point le plus bas de la bosse frontale pour être entraînées par le courant principal sous la nappe d'huile à laquelle elles peuvent éventuellement être incorporées par coalescence. Bien évidemment, toutes les vitesses qui viennent d'être indiquées ne correspondent qu'à des ordres de grandeur. Elles varient avec la densité de l'huile, sa viscosité et la tension interfaciale.

\section{Les ondes d'interface}

Il est établi que les ondes qui parcourent l'interface sont générées par l'instabilité de Kelvin-Helmoltz. Cette instabilité apparaît lorsque deux fluides de densités différentes s'écoulent l'un au-dessus de l'autre. Sans tension interfaciale, l'écoulement est toujours instable. Dans notre cas, on obtient une différence minimale de vitesse nécessaire au déclenchement du mécanisme en fonction du nombre de Weber

soit :

$$
w=2 \frac{2-\Delta}{1-\Delta}
$$

ce qui donne une vitesse de l'ordre de $0,15 \mathrm{~m} / \mathrm{s}$ pour une huile ordinaire. La viscosité de l'huile limite la croissance de ces ondes. C'est à la crête de celles-ci que les gouttelettes sont généralement arrachées dans la zone frontale.

\section{L'onde frontale}

La bosse qui se forme au "bord d'attaque" de la nappe d'huile est créée par le frottement et la pression dynamique, mais il est difficile de définir l'influence respective de l'un et de l'autre.

En négligeant le frottement, on obtient une formule dérivée par Von Karman et qui, depuis, a été largement utilisée pour les fronts de courant de densité (salinité, chaleur...), donc pour des écoulements de fluides miscibles

$$
h=\alpha \frac{U^{2}}{2 g \Delta} \quad \text { avec } \alpha \text { voisin de } 2
$$

Hale, Norton et Rodenberger proposent de compléter la description des phénomènes en jeu en prenant en compte un nombre de Reynolds et un nombre de 


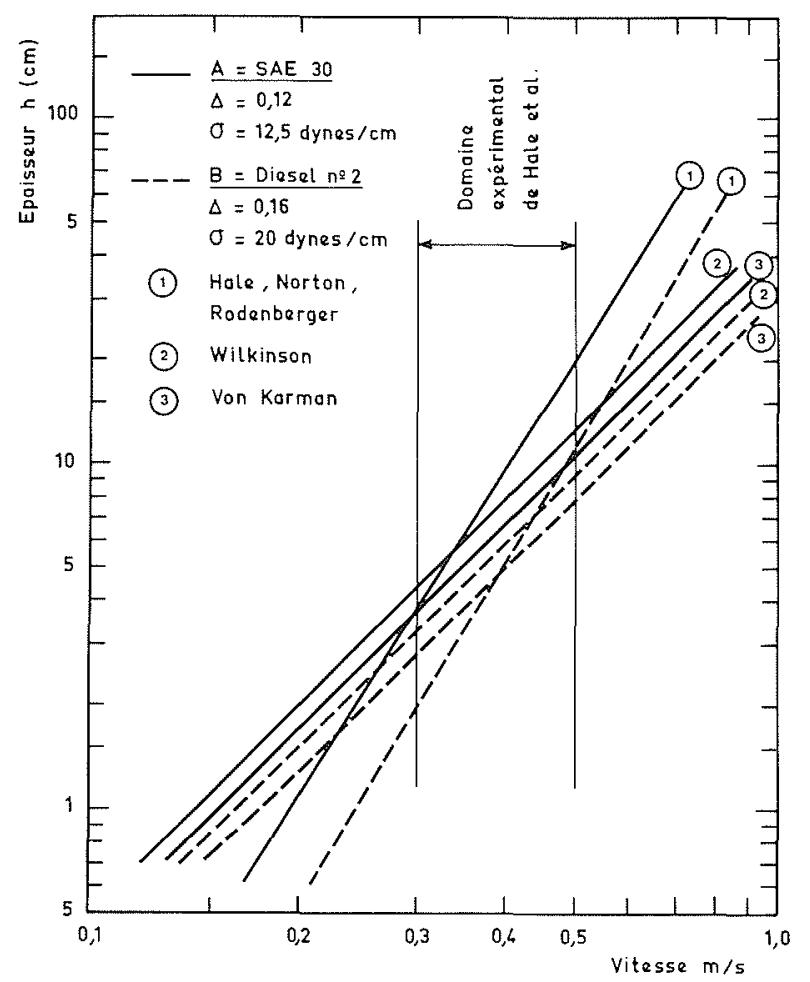

Figure 5 - Comparaison des épaisseurs dans la zone frontale selon différents auteurs.

Weber. Par ajustement à des valeurs expérimentales on obtient :

$$
h=0,595 \frac{U^{3,254}}{g^{1,319} \Delta^{1,319} \sigma^{0,235}}
$$

Mais ces deux critères ne donnent pas d'écarts très nets (figure 5).
Une étude récente (Milgram et Van Houten-figure 6) a proposé de mieux distinguer les effets de pression et de frottement en calculant la pression à partir d'un écoulement potentiel. Ces auteurs mettent en évidence, à faible vitesse, l'importance du frottement sur la forme de la nappe. Par contre à forte vitesse, lorsque la bosse frontale est nettement formée, l'hypothèse de l'écoulement potentiel n'est plus valable comme l'indique la valeur du coefficient de frottement. Les conclusions qu'on peut en tirer sont moins certaines.

En profondeur faible, la bosse frontale devient ins. table (Wilkinson) par un effet d" auto-soutirage", audelà d'une certaine valeur de la vitesse qui peut être exprimée comme un nombre de Froude :

$$
\frac{U}{\sqrt{\Delta g D}}=0,4
$$

\section{L'arrachement}

L'arrachement est le phénomène le plus important à considérer dans la récupération d'hydrocarburé en mer, car il impose une vitesse relative assez faible entre l'eau et l'huile au-delà de laquelle on constate une érosion de la nappe. Les phénomènes mis en jeu sont complexes. Les autres y font intervenir la pression dynamique, la tension interfaciale et la viscosité (Fig. 7).

En étudiant l'équilibre entre la dépression dynamique et la force ascensionnelle de gravité, Cross et Hoult proposent le critère théorique d'instabilité suivant :

$$
\frac{U}{\sqrt{\Delta g h}}>1,4
$$

Ce critère n'est cependant qu'une relation entre $U$ et $h, h$ étant l'épaisseur au maximum de la bosse. Il ne prend pas en compte tous les phénomènes d'interface.
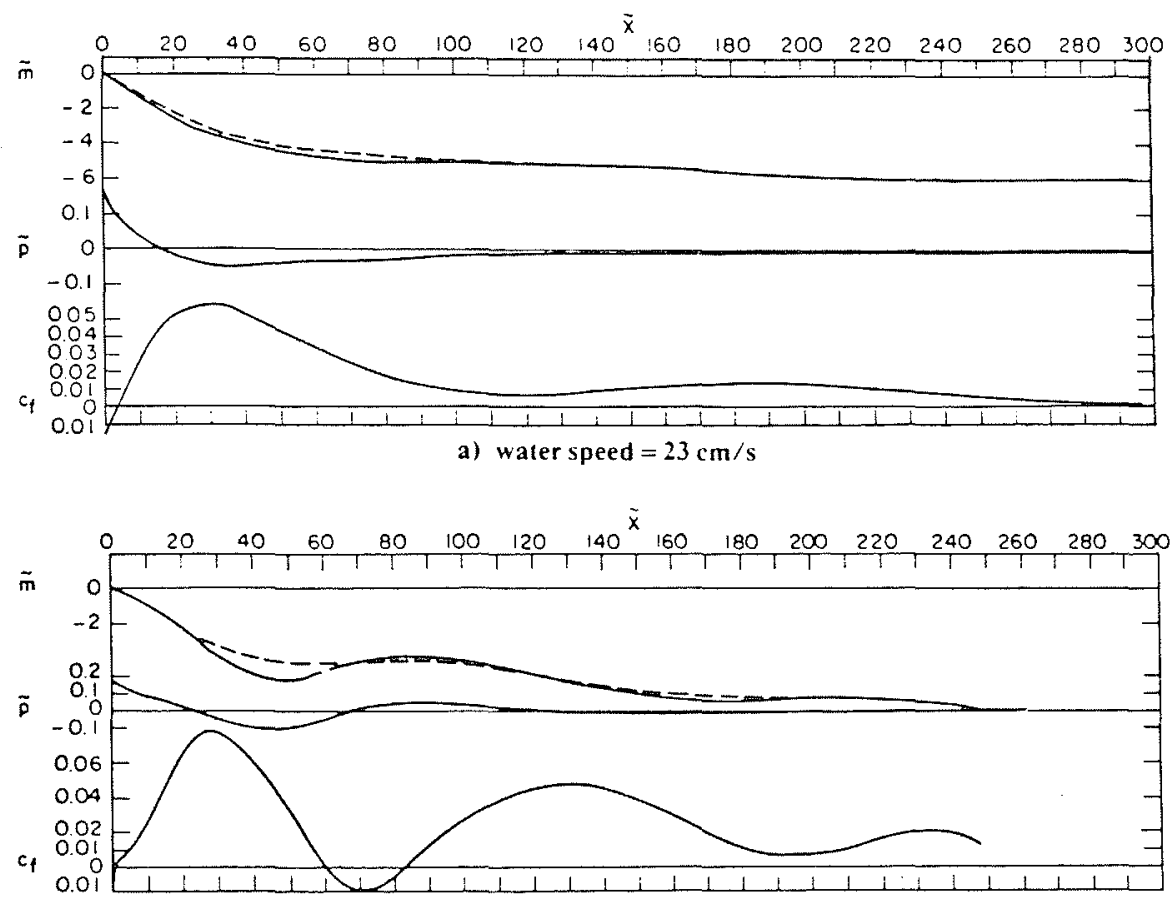

b) water speed $=29 \mathrm{~cm} / \mathrm{s}$

Figure 6 - Profil de nappe - Effet de pression et de frottement (Milgram, Van Houten). 


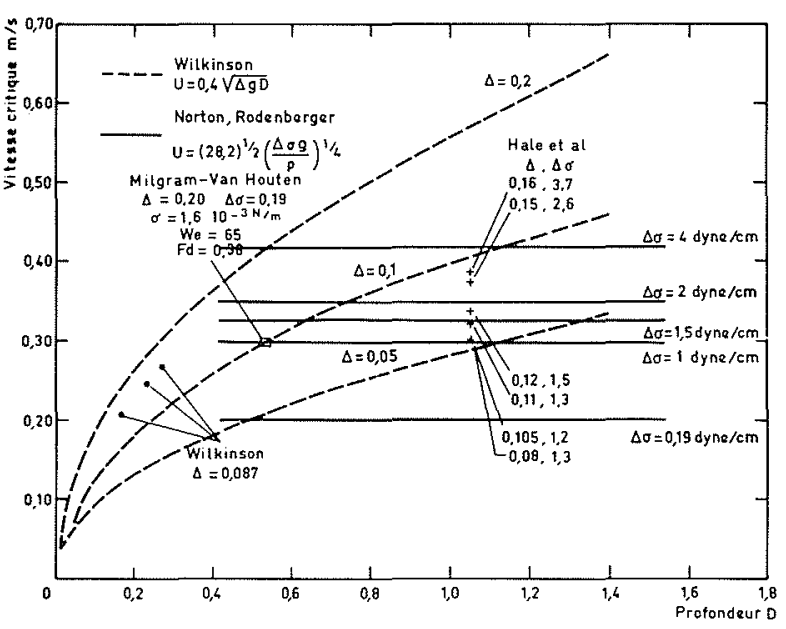

Figure 7 - Stabilité de la zone frontale. Comparaison WilkingonHale, Norton, Rodenberger.

Certains ont proposé d'utiliser plutôt un nombre de Weber critique (Hale, Norton et Rodenberger) ; tel que :

$$
\frac{\rho U^{2}}{\sqrt{\rho \Delta \sigma g}}>28,2
$$

ce qui est ici un véritable critère puisqu'il donne directement $U$.

Cependant, les ajustements de ces critères sur des résultats expérimentaux semblent manquer de généralité, et donc, en dehors d'une limite en vitesse comprise entre $0,30 \mathrm{~m} / \mathrm{s}$ et $0,50 \mathrm{~m} / \mathrm{s}$, il reste difficile de préciser l'écoulement critique au-delà duquel l'arrachement a lieu.

Des essais de quantification du débit d'huile arrachée ont été faits, mais sans grande application pratique jusqu'à présent (Wicks - Lindenmuth - Hale, Norton et Rodenberger).

\section{Une première conclusion}

Pour le comportement d'une nappe d'huile soumise à un cisaillement à l'interface eau-huile, il reste donc, maintenant qu'une description détaillée des phénomènes a été réalisée, à caractériser de manière quantitative l'arrachement d'huile. En raison des conséquences importantes de ce phénomène sur les techniques de lutte anti-pollution, une bonne compréhension de ce phénornène doit permettre de gagner en souplesse d'utilisation des matériels, soit en jouant au mieux des conditions d'environnement, soit en réalisant des matériels mieux adaptés.

\section{Influence des vagues}

L'influence des vagues sur le bicouche eau-huile a été examinée par quelques auteurs, soit à partir d'expériences, soit théoriquement, en fonction de la cambrure des vagues. On note, par rapport à la position dans le courant seul, soit une contraction, soit une élongation de la nappe (Hale, Norton, Rodenberger).

\section{Deux explications}

La première explication théorique consiste à prendre en compte la dérive des particules dans la houle en utilisant les résultats de théories non-linéaires (Madsen). On devrait constater alors une contraction systématique de la nappe, ce qui est en contradiction avec les résultats expérimentaux.

Une analyse plus fine du comportement d'un film à la surface des vagues montre qu'en fonction d'un coefficient adimensionnel lié à la tension dans le film et à la vitesse de propagation des ondes, le film se déplace, soit dans le sens des vagues, soit en sens inverse (Dubeck, Prudhomme). Ce résultat n'a pas été, à notre connaissance, développé pour prendre en compte l'effet d'un courant dans l'eau. Il semble néanmoins correspondre à priori aux expériences.

De manière pratique, il ne semble pas que ce soit là l'aspect le plus important de l'action des vagues pour le problème qui nous intéresse. Néanmoins il soulève un problème théorique intéressant.

\section{ECOULEMENT AUTOUR DES BARRAGES OU VERS LES APPAREILS RECUPERATEURS}

L'écoulement autour des matériels de confinement ou de récupération nous ramène à des domaines d'hydraulique plus traditionnels. On trouve deux points d'intérêt particulier dans cette étude :

- le classique problème des efforts sur un corps, s'appliquant aux barrages;

- l'intéraction du bicouche eau-huile avec l'obstacle, et l'efficacité des barrages.

\section{Une digression : les efforts s'exerçant sur le barrage}

Le problème est d'importance pour ses conséquences pratiques et intéressant d'un point de vue purement théorique. Dans la pratique, ces efforts déterminent directement certaines qualités des matériaux employés dans la construction des barrages (résistance à la traction, au déchircment); ils influencent la conception des matériels (lignes de rappel des efforts, tirant d'eau), et imposent des contraintes dans leur emploi (reprise des efforts, longueur mouillée d'un seul tenant...).

\section{Analyse classique}

Théoriquement, l'analyse des efforts sur un barrage se fait à partir de celle des efforts sur un câble (Fig. 8) dont les composantes élémentaires ont pour équation :

$$
\begin{aligned}
& Q=\frac{1}{2} C_{N}^{N} \rho U^{2} d \cos ^{2} \phi \\
& P=\frac{1}{2} C_{T} \rho U^{2} d \sin \phi
\end{aligned}
$$

On définit des coefficients de traînée élémentaire dans le courant seul. Leur loi de variation avec l'angle d'incidence du courant $\left(\cos ^{2}\right)$ (Cross et Hoult) donne pour 


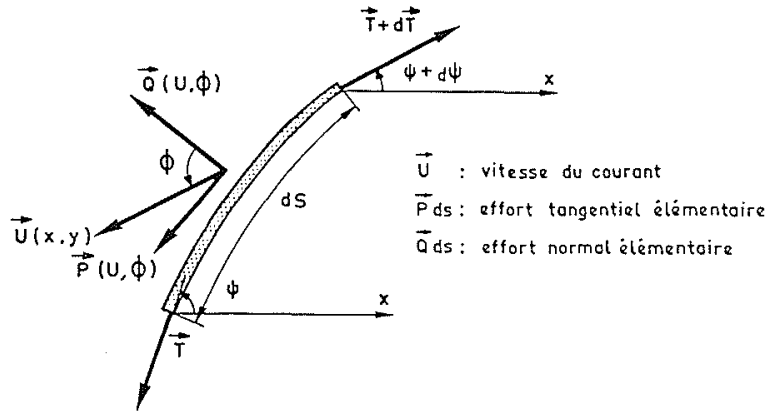

Figure 8 - Efforts élémentaires s'exerçant sur un barrage.

un barrage complet une forme de chaînette, si l'effort tangentiel est négligé.

\section{Des écarts expérimentaux}

Si à cette analyse correspondent de bons résultats expérimentaux lorsque l'incidence est quasi frontale, par contre pour une incidence franchement oblique (barrage en déviation), on constate des écarts importants avec la loi de variation de l'effort élémentaire proposée (Donnars, Hauguel), (Fig. 9), même en prenant en compte un effort tangentiel.

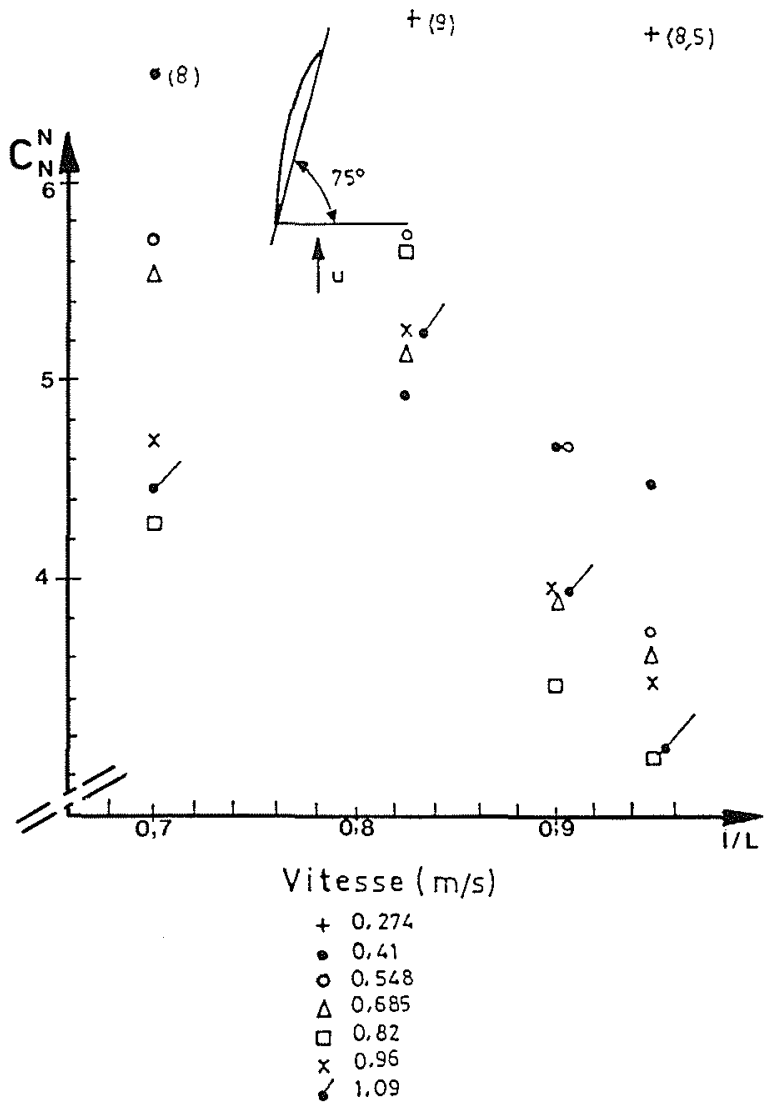

Figure 9 - Variation du coefficient d'effort normal en fonction du coefficient d'ouverture (Donnars, Hauguel).

\section{Influence des autres phénomènes}

En présence d'huile, le coefficient d'effort varie avec la quantité d'huile retenue, cette dernière servant dans un premier temps à profiler le barrage; puis, pour une longueur plus importante, l'effet du frottement sur la nappe l'emporte et le coefficient de traînée augmente (Nielsen).

L'effort dans les vagues est calculé à partir d'un coefficient de traînée empirique, proportionnel au coefficient de traînée en courant seul. Il est fonction de la hauteur de vague, de la période, et de la vitesse du courant principal, mais cette méthode de calcul reste très empirique.

Pour le calcul des efforts, on peut penser que des études hydrodynamiques permettront de disposer d'une modélisation plus satisfaisante des efforts sur les barrages, particulièrement pour leur emploi, en dérivation, qui contrairement à leur nom-barrage - est leur emploi principal.

\section{Retour au sujet : l'écoulement}

L'écoulement du bicouche eau-huile au voisinage d'un barrage, ou d'un système récupérateur, détermine l'efficacité de celui-ci (en excluant les problèmes d'arrachement dans la zone frontale).

\section{Le soutirage}

Cet écoulement montre tout d'abord un aspect classique des écoulements stratifiés : le soutirage. Dans le cas de barrages, il semble que les formules traditionnelles conviennent, avec quelques aménagements des coefficients empiriques.

Par exemple, pour une nappe d'huile arrêtée par un barrage, on retrouve une forme d'écoulement stratifié voisine de celle étudiée par Harleman, Goock et Ippen (Fig. 10).

Leur formule de début de soutirage s'écrit :

$$
\frac{Q^{2}}{\Delta g(D-h)^{3}}=0,25\left\{1-\left(\frac{D-d}{D-h}\right)^{3}\right\}
$$

En profondeur infinie, on obtient une hauteur de barrage $d$ pour retenir une nappe d'épaisseur $h$, telle que :

$$
d=h+\frac{U^{2}}{0,75 \Delta g}
$$

\section{Prise en compte de la profondeur}

En profondeur limitée, il est nécessaire de connaître $h$ à l'amont du barrage. On peut utiliser la valeur de Cross et Hoult. On obtient alors la relation suivante pour la hauteur du barrage :

$$
\begin{gathered}
\frac{d}{D}=1-\left\{1-C_{f}^{1 / 2} F_{w} \frac{x^{1 / 2}}{D}-\frac{1}{0,25} F_{w}^{2}\right\}^{1 / 3} \\
\text { avec } \\
F_{w}=\frac{U}{\sqrt{\Delta g D}}
\end{gathered}
$$

Si $d=D$ le soutirage ne peut être empêché, ce qui se produit pour

$$
\frac{h}{D}=1-1,59 \frac{U^{2 / 3}}{\sqrt{\Delta g D}}
$$




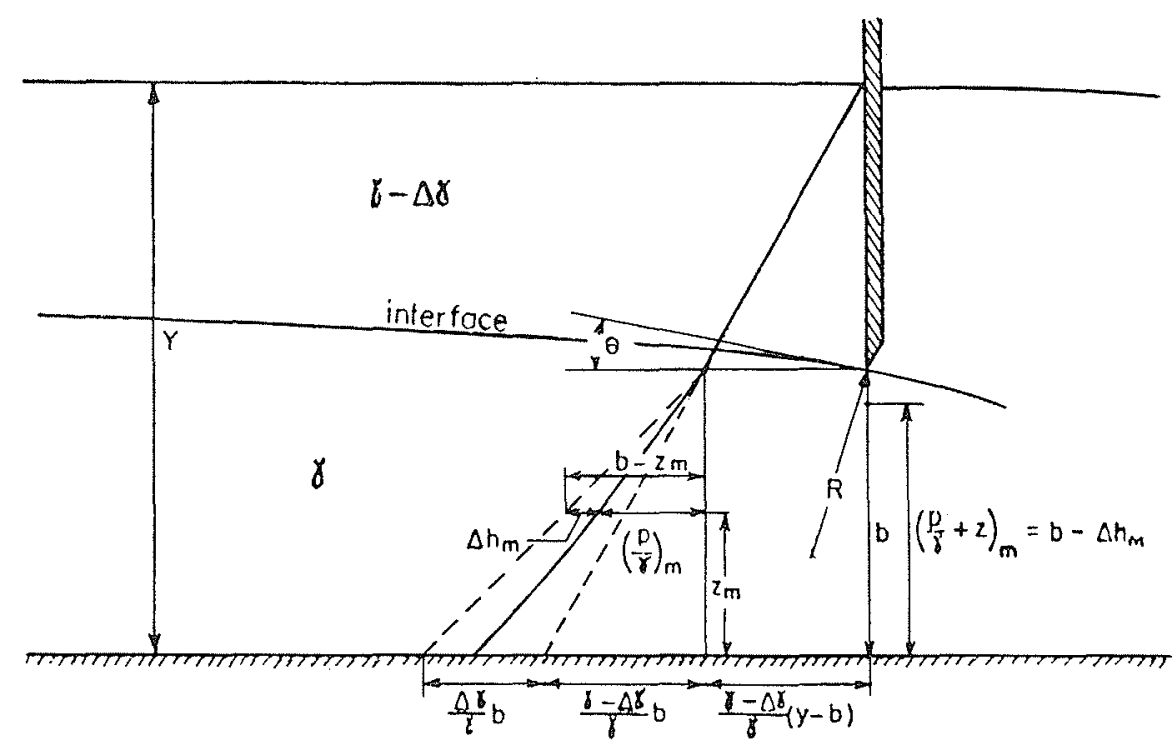

Figure 10 - Ecoulement sous une vanne (Harleman, Goock, Ippen).

On retrouve ici la formule de limite d'épaisseur de Wilkinson au coefficient expérimental d'Harleman près $(1,59)$. Ce qui prouve bien la validité des formules classiques de soutirage.

Deux autres aspects sont plus spécifiques du problème traité ici :

- le franchissement du barrage par les ondes d'interface ;

- l'entraînement d'huile par les tourbillons.

\section{Influence des ondes d'interface}

Lorsque les ondes d'interface arrivent sur le barrage, elles s'y réfléchissent, créant ainsi une agitation importante juste à l'amont de l'obstacle. Dans certains cas, lorsque la couche d'huile est suffisamment épaisse, on assiste au franchissement et au passage d'huile derrière le barrage. L'agitation elle-même peut amener à la création de gouttes d'huile qui seront entraînées par l'écoulement principal.

\section{Les tourbillons}

De plus, autour de lobstacle, particulièrement lorsque l'incidence n'est pas frontale, des tourbillons se créent. Ils entraînement l'huile dès qu'ils sont suffisamment forts pour morceler la nappe, ce qui se produit presque dès l'apparition de ceux-ci. L'apparition des tourbillons peut être fortement modifiée par les vagues dans la mesure où elles perturbent fortement l'écoulement de l'eau.

Ces deux phénomènes sont pris en compte de manière uniquement empirique par les utilisateurs de systèmes de récupération, et très peu par les concepteurs dans la mesure où ils sont difficiles à évaluer a priori, bien que certaines études aient eu lieu particulièrement sur les barrages en déviation (Mac Cracken).

\section{UN RESUME}

Avant de tirer des conclusions, résumons rapidement nos connaissances.

La nappe d'huile se constitue essentiellement sous l'effet du frottement à l'interface eau-huile. Cette interface peut être instable sous l'effet de mécanismes encore connus de manière imprécise, mais à partir de vitesses assez faibles $(0,40 \mathrm{~m} / \mathrm{s})$.

Pour un barrage, on obtient schématiquement trois types de défaillance :

- débordement par remplissage du barrage et soutirage ; - entraînement de gouttelettes après "déferlement" des ondes d'interfaces;

- arrachement de gouttelettes dans la zone frontale.

$A$ ces phénomènes mettant en cause essentiellement la mécanique de la nappe d'hydrocarbures, il faut ajouter l'arrachement par des tourbillons créés par les décollements de l'écoulement autour du barrage, et celui dû aux protubérances du barrage. Pour ceux-ci le comportement marin du barrage est un paramètre essentiel.

D'une manière générale, ces caractéristiques s'appliquent aux systèmes de récupération, mais les écoulements qu'ils engendrent lors du pompage compliquent ce schéma.

\section{DES CONCLUSIONS}

\section{Points de recherche dégagés}

En conclusion de ce rapide panorama de l'hydraulique et de la récupération d'hydrocarbures en mer, il nous semble que la recherche hydraulique après avoir réussi dans la décennie passée à donner une image des principaux phénomènes en jeu, devra s'attacher à résoudre les points suivants, pour les systèmes de confinement et de récupération : 


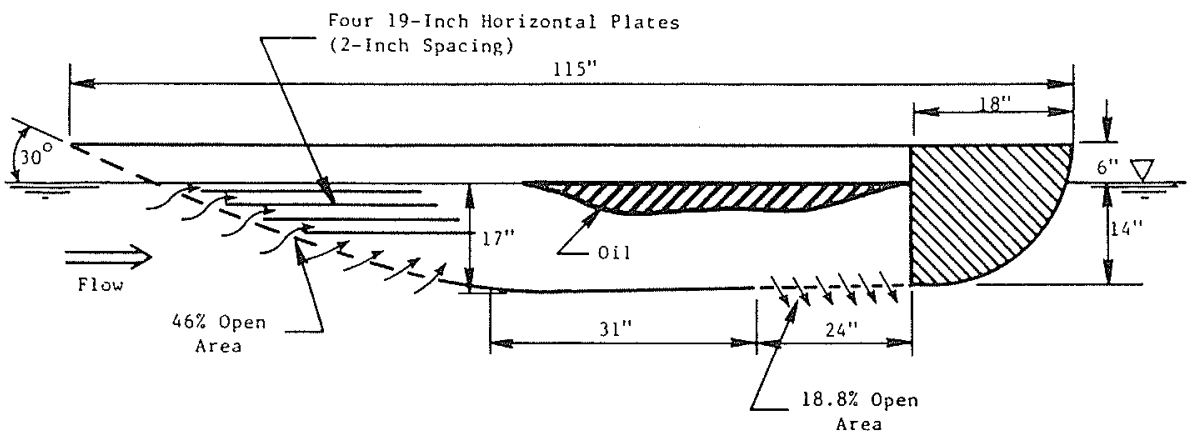

Figure 11 - Barrage avec perte de charge et profil double (Ayers).

- contrôler ou inhiber l'arrachement afin d'augmenter la plage d'emploi de ces matériels ;

- estimer l'influence des vagues sur ces phénomènes; - évaluer les efforts s'exerçant sur un barrage flottant pour des angles d'incidence faible.

\section{Développements en cours}

Il est d'à propos de citer ici un certain nombre de recherches, en cours ou passées, qui portent sur le perfectionnement du système barrage récupérateur. On peut citer les barrages doubles et profilés (Wilcox, Ayers. . .), des systèmes avec perte de charge (Ayers, Jensen et al, NHL, ... ) (Fig. 11).

Dans une autre direction, on peut retenir les systèmes à jet d'eau pour contrôler la forme de la nappe (Fig. 12).

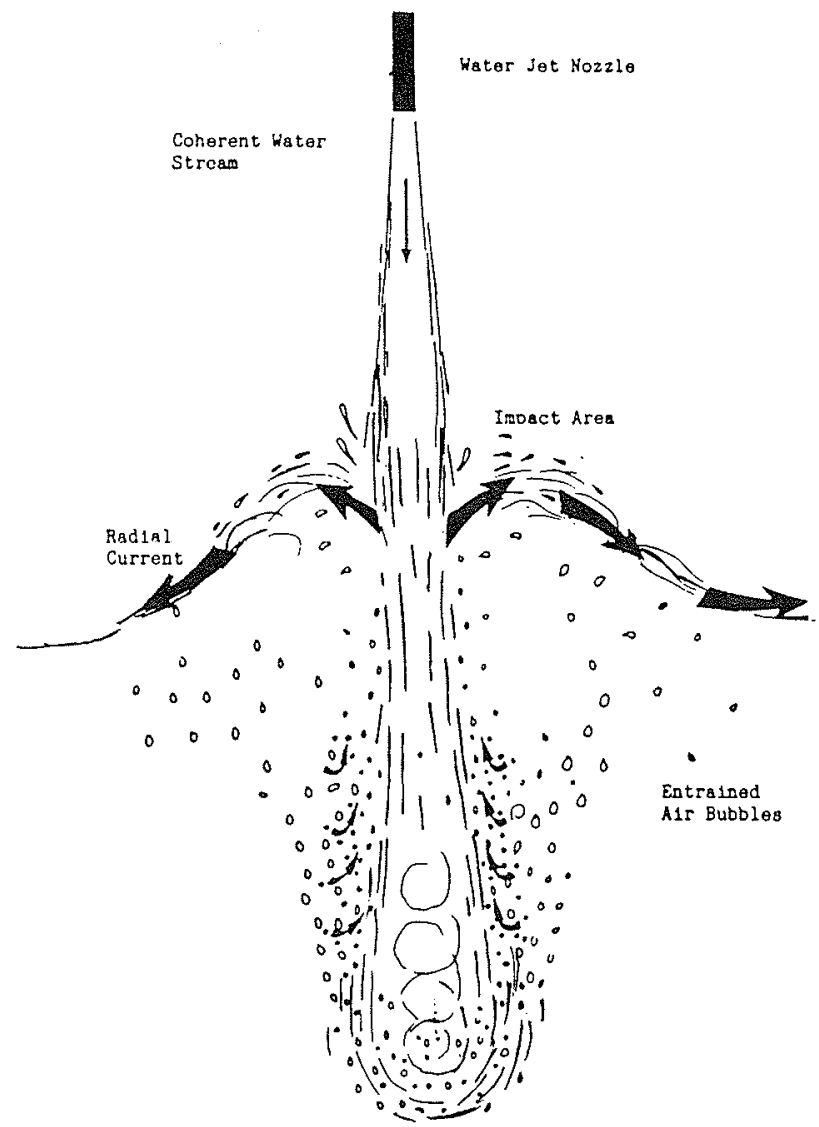

Figure 12 - Effet d'un jet de surface (Nash, Johnson).
Ces techniques nouvelles à leur tour soulevent un certain nombre de problèmes hydrauliques, qui sont résolus de manière relativement empirique, mais qui sont intéressants d'un point de vue théorique.

Les résultats de ces recherches devront s'intégrer bien évidemment dans des études de prototypes de système de récupération et elles devront prendre en compte toutes les contraintes technologiques qui en découlent (technologie des matériaux, maniabilité des systèmes....) .

\section{Nécessité de méthodologies de récupération}

Enfin une dernière phrase pour rappeler que si bien des voies nouvelles existent, il n'en demeure pas moins que des limites très restrictives resteront quant à l'efficacité des systèmes de confinement et đe récupération des hydrocarbures. Il est donc nécessaire de développer en parallèle des méthodologies de récupération, particulièrement à la côte où les problèmes sont plus critiques - faune et flore abondantes, marge de manœuve faible particulièrement en mer à marée et dans les zones marécageuses - et d'appliquer ces méthodologies en préparant à l'avance des dossiers d'intervention. Au large une action dynamique de récupération doit être menée; elle nécessite encore bien des développements des systèmes de récupération et de confinement.

\section{Notations}

$g \quad$ accélération de la gravité

$U \quad$ vitesse de l'eau

$h \quad$ épaisseur d'huile

$C_{f} \quad$ coefficient de frottement

$\Delta \quad$ écart relatif de densité $\left(\Delta=\frac{\rho \text { eau }-\rho \text { huile }}{\rho \text { eau }}\right)$

$x \quad$ longueur de la nappe

$f, f_{0} \quad$ coefficient de frottement (Wilkinson)

$F_{w} \quad$ nombre de Froude utilisé par Wilkinson

$F_{w}=\frac{U}{\sqrt{g \Delta D}}$

$D \quad$ profondeur d'eau

$W \quad$ nombre de Weber $\left(W=\frac{\rho U^{2}}{\sqrt{\rho \sigma \Delta g}}\right)$ 
o tension interfaciale eau-huile

$P, Q \quad$ efforts élémentaires s'exerçant sur un barrage (Fig. 8)

$C_{N}^{N} \cdot C_{T}$ coefficients adimensionnels d'effort.

\section{Bibliographie}

Nota : La bibliographie ci-dessous ne cherche pas à être exhaustive. Elle présente différents aspects de l'hydraulique des systèmes de confinement et de récupération d'hydrocarbures.

AGRAWAL R.K., HALE L.A. - A new criterion for predicting headwave instability of an oil slick retained by a barrier. OTC, 1983 - pp. 461-464, Offshore Technology Conference 1974.

AYERS R.R. - A rigid perforated plate oil boom for high currents. Environmental Protection Technology Series. EPA 600/2/76-263, December 1976.

BROWN G.A. - In-situ measurements of oil barriers shape and loads due to current action. Proc. Donf. on Prevention. Control of oil spills. EPA-API-USCG Washington, Decembe $1973,409.419$.

CHANDRASEKHAR S. - Hydrodynamic and Hydromagnetic stability. Cambridge at the University Press.

CRAYA A. - Recherches théoriques sur l'écoulement des couches superposées de fluides de densités différentes. La Houille Blanche, janvier-février 1949.

CROSS R.H., HOULT D.P. - Oil Booms in Tidal Currents. Coastal Engineering, 1970, pp. 1754-1759.

CROSS R.H., HOULT D.P. - Collections of oil slicks. $J$. Waterways, Harbour and Coastal Engg. Div. Proc. A.S.C.E., May, 1971, 313-322.

DONNARS Ph., HAUGUEL A. - Effet d'échelle lors de l'étude expérimentale de la défaillance d'un barrage antihydrocarbures. Rapport Electricité de France. $D E R$ $H E 42 / 80.28,1980$.

DONNARS Ph., HAUGUEL A. Barrages flottants anti-hydrocarbures. Etude expérimentale du comportement d'un barrage soumis à un courant uniforme. Rapport Electricité de France. DER HE42/80.57.

DUDECK $M$, PRUD'HOMME R. Vitesse de glissement d'un film sur la surface d'un liquide avec onde de gravité. C.R. Acad. Sc. Paris t. 282, 21 juin 1976. Série B 523-526.

ERYUZLU N.E., HAUSSER R. - Use of floating deflectors for oil spill control infast flowing water. Oil Spill Conference. EPA-API-USCG, New-Orleans, 1977, 335-340.

HALE L.A., NORTON D.J., RODENBERGER C.A. - The effects of currents and waves on an oil slick retained by a barrier. Rapport GG-D-53-75, Texas $A$ \& $M$, Research Foundation, April 1974.

HARLEMAN D.R.F., GOOCK R.S., IPPEN A.T. - Submerged sluice control of stratified flow. Proc. A.S.C.E., J. of Hyd. Div., avril 1958, paper 1584 .

JENSEN D.S., LINDENMUTH W., BEACH R.L., NORTON D.J. - Energy dissipative devices to control oil slicks in fast current environments. Proc. Conf. Prevention and
Control of oil pollution. EPA-API-USCG, San-Francisco Cal., March 1975, 355-364.

LARRABEE R.M., BROWN G.A. - An in-situ investigation of oil barrier shape and drag coefficients. Rapport CG-D-161-75, July 1974.

LEHR W.E., SCHERER J.O. - Design requirements for booms. Proc. Joint Conf. on prevention and Control of oil spills. APIFWPCA. New-York, 1969, 107-128.

LEPETIT J.P., DARRAS M. - Barrages flottants anti-hydrocarbures. Rapport $\mathrm{n}^{\circ} 1$. Etude bibliographique du comportement de la nappe d'huile. Rapport Electricité de France DER HE042/78-24, 1978

LINDENMUTH W.T., MLLER E.R., HSU C.C. - Studies of oil retention boom hydrodynamics. Hydronautics Inc., Laurel, Maryland, December 1970.

LINDENMUTH W.T., SHERER I.O., VANDYKE P. - Analysis and model tests to determine Forces and Motions of an Oil Retention Boom. Technical Report 93B-1 Hydronautics. Laurel, Maryland-AD-202-512/AD 702513. 1970.

MAC CRACKEN W.E. - Hydrodynamics of diversionary booms. Environmental Protection Technology series. EPA 600/2/ 78-075, April 1978.

MILGRAM J.H., O'DEA J.F. - Evaluation of the strength and seakeeping ability of pollution control barriers. MIT Rapport CG-D-55-75, September 1974.

MILGRAM J.H. Physical requirements for oil pollution control barriers. Proc. Conf. on Prevention. Control of oil spills. EPA-API-USCG Washington, December 1973, 375 381.

MILGRAM J.H. VAN HOUTEN R.J. - Mechanics at a restrained layer of floating oil above a water current. $J$. of Hydronautics. Vol. $12 \mathrm{~N}^{\circ} 3$, July 1978, pp.93-108.

NASH J.H., JOHNSON M.G. - Coherant plunging water jets for oil spills control. Oil spill conference, 1981. 655-660.

NIELSEN F.G. - Oil barriers in two-dimensional stationary flow. Norwegian Maritime Research $N^{\circ} 2,1978$, pp. 2.13.

ROBBINS R.E. - The Oil Boom in a Current. M.S. Thesis, Department of Electrical Engineering, Massachusetts Insti tute of Technology, 1970.

STOLZENBACH, MADSEN, ADAMS, POLLACK, COOPER. $A$ review and evaluation of basic techniques for predicting the behaviour of surface of oil slicks. M.I.T., Rapport M.I.T.$S G 77.8$, mars 1977 .

WICKS M. - Fluid Dynamics of floating oil containment by Mechanical Barriers in the presence of Water Currents. Proc. Joint Conf. on Prevention and Control of oil Spills, Am. Petroleum Inst., December 1969, pp. 55-106.

WILCOX J.D. - A hydrodynamically effective horizontal oil boom. Proc. Conf. Prevention and Control of oil Pollution, 1975.

WILKINSON D.L. - Containment of oil slicks in the St. Lawrence river. Rapport LTR-HY-16, Div. Génie Mécanique, Ottawa, Canada, December 1971.

WILKINSON D.L. - Dynamics of contained oil slicks. $J$. of Hydraulics Div., Proc. A.S.C.E., June 72, pp. 1013-1030.

WILKINSON D.L. - Collection of Oil Slicks. Discussion. J of Waterways, Harbours and Coastal Engg. Div. Proc. A.S.C.E., February 72 , pp. 122-124.

WILKINSON D.L. - Limitation to length of contained oil slicks. Proc. A.S.C.E.-HY 5, May 1973, pp. 701-712. 